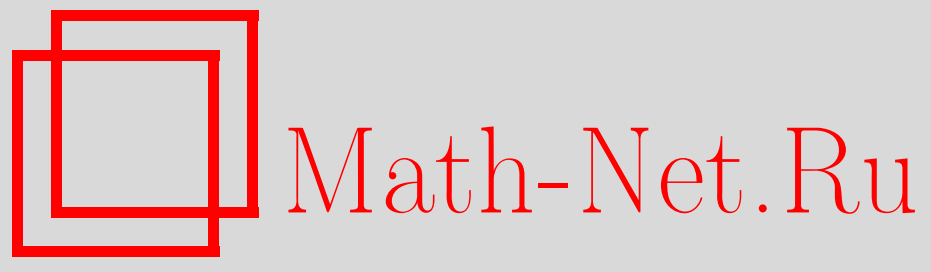

А. В. Фонарёв, Исключительные векторные расслоения на грассманианах, УМH, 2014, том 69, выпуск 4, 189-190 DOI: https://doi.org/10.4213/rm9609

Использование Общероссийского математического портала Math-Net.Ru подразумевает, что вы прочитали и согласны с пользовательским соглашением http: //www . mathnet.ru/rus/agreement

Параметры загрузки:

IP : 54.80 .73 .141

26 апреля 2023 г., $11: 47: 16$

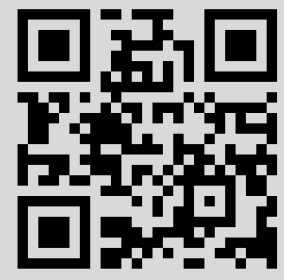




\section{Исключительные векторные расслоения на грассманианах}

\section{А. В. Фонарёв}

Пусть $X=\operatorname{Gr}(k, V)$ - грассманиан $k$-мерных подпространств в векторном пространстве $V$ размерности $n$. Обозначим через $U$ тавтологическое расслоение. В частности, на $X$ имеется короткая точная последовательность

$$
0 \rightarrow U \rightarrow V \otimes \mathscr{O}_{X} \rightarrow V / U \rightarrow 0 .
$$

Для всякой диаграммы Юнга $\alpha$ можно построить эквивариантные векторные расслоения $\Sigma^{\alpha} U$ и $\Sigma^{\alpha}(V / U)$, применив соответствующий функтор Шура $\Sigma^{\alpha}$. Обозначим через $\mathrm{Y}_{a, b}$ множество диаграмм, вписанных в прямоугольник размера $a \times b$.

Пусть $\gamma$ - строго монотонный путь на вещественной плоскости, ведущий из точки $(0,0)$ в точку $(n-k, k)$. Рассмотрим точки $\gamma$ по меньшей мере с одной целочисленной координатой: $(0,0)=\left(x_{0}, y_{0}\right),\left(x_{1}, y_{1}\right), \ldots,\left(x_{l-1}, y_{l-1}\right),\left(x_{l}, y_{l}\right)=(n-k, k)$ в порядке возрастания первой координаты.

Данная работа посвящена доказательству следующей теоремы, предложенной в качестве гипотезы в [1] (гипотеза 9.8).

Теорема. Имеется полуортогоналъное разложение ограниченной производной категории грассманиана:

$$
D^{b}(X)=\left\langle\mathscr{A}_{0}, \mathscr{A}_{1}(1), \ldots, \mathscr{A}_{l}(l)\right\rangle,
$$

где $\mathscr{A}_{i}$ порождается векторными расслоениями $\Sigma^{\lambda} U^{*} \otimes \Sigma^{\mu}(V / U)$ для всех возможных $\operatorname{nap}(\lambda, \mu) \in \mathrm{Y}_{y_{i}, n-k-x_{i}} \times \mathrm{Y}_{x_{i}, k-y_{i}}$.

Для начала опишем строение подкатегорий $\mathscr{A}_{i}$. Зафиксируем пару чисел $0 \leqslant a \leqslant k$, $0 \leqslant b \leqslant n-k$ и пару диаграмм $\left(\lambda_{0}, \mu_{0}\right) \in \mathrm{Y}_{a, b} \times \mathrm{Y}_{n-k-b, k-a}$. Прямая проверка с применением теоремы Бореля-Ботта-Вейля [2] и правила Литтлвуда-Ричардсона [3; p. 121] показывает следующее.

ПредЛОЖЕНИЕ 1. Множество расслоений

$$
\left\{\Sigma^{\lambda} U^{*} \otimes \Sigma^{\mu}(V / U) \mid \lambda \subseteq \lambda_{0}, \mu \subseteq \mu_{0}\right\}
$$

образует исключительный блок в смысле [1].

Как следствие, если в эквивариантной категории $D_{G}^{b}(X)$ перейти к двойственному исключительному набору $\left\{\mathscr{E}_{\lambda, \mu}\right\}_{\lambda \subseteq \lambda_{0}, \mu \subseteq \mu_{0}}$, он, в свою очередь, окажется исключительным уже в обычной производной категории $D^{b}(X)$. Более того, можно проверить, что $\mathscr{E}_{\lambda, \mu}$ не зависит от выбора диаграмм $\lambda_{0}$ и $\mu_{0}$.

Рассмотрим многообразие частичных флагов $Y=\mathrm{Fl}(k, n-b ; V)$ вместе с проекциями

$$
\operatorname{Gr}(k, V)=X \stackrel{p}{\longleftarrow} Y \stackrel{q}{\rightarrow} \operatorname{Gr}(n-b, V) .
$$

Обозначим через $W$ и $U$ тавтологические подрасслоения $U \subset W \subset V \otimes \mathscr{O}_{Y}$ на $Y$ рангов $k$ и $n-b$ соответственно. Пусть $(\lambda, \mu) \in \mathrm{Y}_{a, b} \times \mathrm{Y}_{n-k-b, k-a}$. Прямым вычислением с использованием формулы проекции последовательно для отображений $p$ и $q$ получаем следующее утверждение.

ПреДлОЖениЕ 2. Имеем $\mathscr{E}_{\lambda, \mu}^{*}=p_{*}\left(\Sigma^{\lambda} W \otimes \Sigma^{\mu}(W / U)^{*}\right)$, причем высшие прямые образы зануляются: $R^{i} p_{*}\left(\Sigma^{\lambda} W \otimes \Sigma^{\mu}(W / U)^{*}\right)=0$ при $i>0$.

Предположим, что $\lambda$ имеет максимально возможную ширину $b$. Тогда выполнено следующее.

Работа выполнена при поддержке Лаборатории алгебраической геометрии НИУ "ВШЭ" (грант Правительства РФ, дог. 11.G34.31.0023) и фонда Д. Зимина “Династия".

DOI: $10.4213 / \mathrm{rm} 9609$ 
ПреДЛОЖенИЕ 3. Для некоторых челых чисел $0<n_{1}<n_{2}<\cdots<n_{b}<n$ и диаграмм $\lambda_{b} \subset \lambda_{b-1} \subset \cdots \subset \lambda_{1} \subset \lambda$ имеется точная последовательность вида

$$
0 \rightarrow \mathscr{E}_{\lambda^{-}, \mu^{+}}(-1) \rightarrow \mathscr{E}_{\lambda_{b}, \mu} \otimes \Lambda^{n_{b}} V^{*} \rightarrow \cdots \rightarrow \mathscr{E}_{\lambda_{1}, \mu} \otimes \Lambda^{n_{1}} V^{*} \rightarrow \mathscr{E}_{\lambda, \mu} \rightarrow 0
$$

где $\lambda^{-}$получается из $\lambda$ удалением первой строки, $\mu^{+}$получается из $\mu$ добавлением первого столбца высоты $n-k-b$, а числа $n_{i}$ u диаграммы $\lambda_{j}$ такие же, как в [4; предложение 5.3].

Для доказательства предложения 3 нужно рассмотреть соответствующие комплексы на $\operatorname{Gr}(n-b, V)$, построенные в работе [4], и применить к ним преобразование Фурье-Мукаи $p_{*}\left(\Sigma^{\mu}(W / U)^{*} \otimes q^{*}(-)\right)$.

НАБРОСОК ДОКАЗАТЕЛЬСТВА ТЕОРЕМЫ. ЗаметИМ, чТО ЛЮбой строГо монотонНЫЙ путь из точки с координатами $(0,0)$ в точку с координатами $(n-k, k)$ можно продеформировать в любой другой. При этом подкатегории $\mathscr{A}_{i}$ меняются только при проходе пути через точку целочисленной решетки.

Заметим, что пути, идущему сначала вверх, а затем вправо, соответствует разложение, получающееся небольшой перестройкой полного исключительного набора, построенного М. Капрановым в [5]. Значит, достаточно проверить, что при элементарных преобразованиях вида

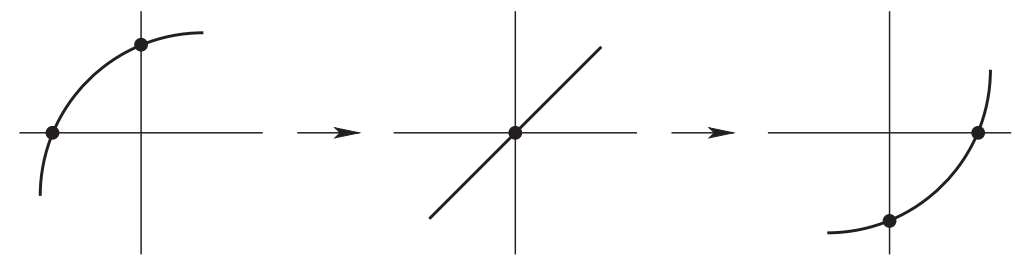

происходит перестройка полуортогонального разложения.

Оказывается, можно ограничиться преобразованиями вида

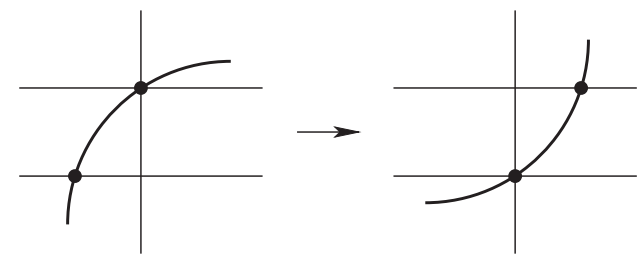

которые, в свою очередь, явно описываются комплексами типа (2).

Следствие. Рассмотрим $X=\mathrm{Gr}(k, 2 k)$, а в качестве пути $\gamma$ возвмем прямолинейный отрезок, соединяющий точки $(0,0)$ u $(k, k)$. Соответствующее полуортогональное разложение инвариантно относительно внешнего автоморфизма $X$.

\section{Список литературы}

[1] A. Kuznetsov, A. Polishchuk, "Exceptional collections on isotropic Grassmannians", J. Eur. Math. Soc. (to appear); 2011, 51 pp., arXiv: 1110.5607. [2] M. Demazure, Invent. Math., 33:3 (1976), 271-272. [3] W. Fulton, Young tableaux. With applications to representation theory and geometry, Cambridge Univ. Press, Cambridge, 1997. [4] А. В. Фонарёв, Изв. РАН. Сер. матем., 77:5 (2013), 203-224. [5] M. M. Kapranov, Invent. Math., 92:3 (1988), 479-508.

Антон Вячеславович Фонарёв (Anton V. Fonarev)

Математический институт им. В. А. Стеклова РАН; НИУ "ВШЭ"

E-mail: avfonarev@mi.ras.ru
Представлено Д. О. Орловым Принято редколлегией 23.06.2014 